\title{
A Self-Boost Charge Pump Topology for a Gate Drive High-Side Power Supply
}

\author{
Shihong Park, Student Member, IEEE, and Thomas M. Jahns, Fellow, IEEE
}

\begin{abstract}
A self-boost charge pump topology is presented for a floating high-side gate drive power supply that features high voltage and current capabilities for use in integrated power electronic modules (IPEMs). The transformerless topology uses a small capacitor to transfer energy to the high-side switch from a single power supply referred to the negative rail. Unlike conventional bootstrap power supplies, no switching of the main phase-leg switches is required to provide power continuously to the high-side gate drive, even if the high-side switch is permanently on. Additional advantages include low parts-count and simple control requirements. A piecewise linear model of the self-boost charge pump is derived and the circuit's operating characteristics are analyzed. Simulation and experimental results are provided to verify the desired operation of the new charge pump circuit. Guidelines are provided to assist with circuit component selection in new applications.
\end{abstract}

Index Terms-Bootstrap power supply, floating, high-side gate drive, MOS-gated power semiconductors, power module, transformerless.

\section{INTRODUCTION}

\section{A. Background}

B OOTSTRAP circuits are widely used in bridge inverters to provide the floating power supply for high-side switch gate drives [1]. They are often preferred over high-frequency transformer circuits due to their simplicity and basic compatibility with integrated circuit implementation, making them well suited for achieving low cost and high reliability.

However, the bootstrap technique imposes significant limitations due to its periodic charging time requirements that can interfere with the desired gate drive operation under some important operating conditions [1]. In particular, the high-side gate drive can become starved of energy if the inverter switching algorithm calls for either switch to be on for long intervals. Under such circumstances, the main phase-leg switch may be forced to perform a brief switching cycle in order to refresh the depleted capacitor charge for the high-side gate drive.

One approach that has been proposed to overcome these problems uses an auxiliary charge pump [2]-[4]. This technique can

Manuscript received March 31, 2003; revised August 18, 2004. This work was supported by the ERC Program of the U.S. National Science Foundation under Award EEC-9731677 and by the Wisconsin Electric Machines and Power Electronics Consortium (WEMPEC). Recommended by Associate Editor F. Blaabjerg.

S. Park is with the School of Electrical, Electronics, and Computer Engineering, Dankook University, Seoul 140-714, Korea (e-mail: shihong@ dankook.ac.kr).

T. M. Jahns is with the University of Wisconsin-Madison, Madison, WI 53706 USA (e-mail: t.jahns@ieee.org).

Digital Object Identifier 10.1109/TPEL.2004.843013

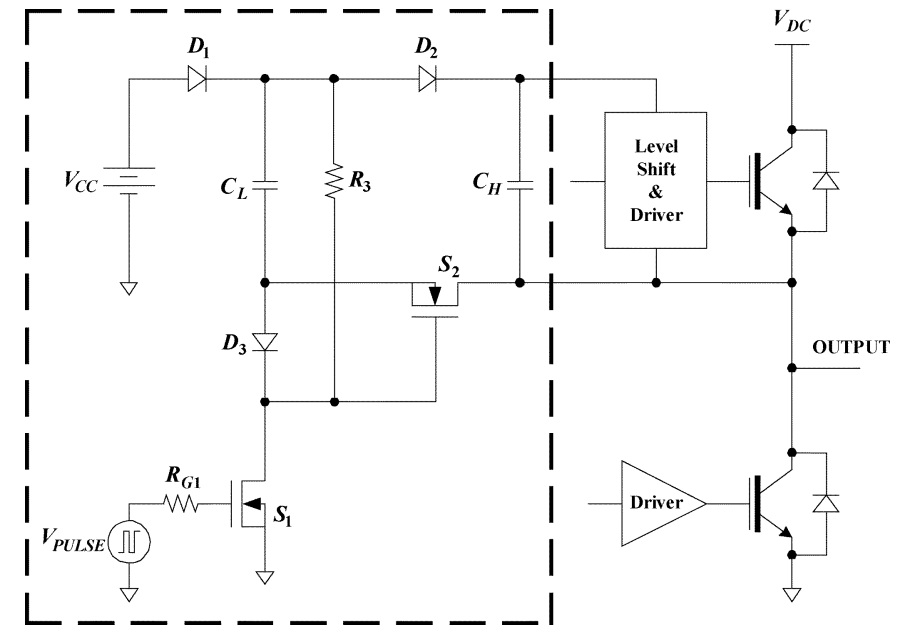

Fig. 1. Self-boost charge pump circuit configuration for an inverter phase leg high-side power supply.

provide the needed power for a high-side gate drive without interfering with the desired phase-leg switching sequence. However, it is not widely used due to its higher complexity and its requirements for high-voltage level shifting to supply control signals to the auxiliary switches.

Another technique has been described in the literature [5], [6] that applies charge pump techniques to deliver power to a floating high-side power supply. It has a simple topology and overcomes the limitation of conventional bootstrap high-side power supplies by transferring charge even when the upper switch is permanently on. However, this technique suffers in high current applications from high power losses in the charge pump oscillator and high voltage ripple due to large voltage surges caused by switching of the main phase-leg switches. These disadvantages limit this technique to gate drive applications with low current supply requirements.

A new charge pump topology is presented in this paper for floating high-side gate drive power supplies that avoid the problems of existing techniques while retaining the advantages of simplicity and compatibility with integrated circuit implementation. This topology, shown in Fig. 1 with a standard inverter phase leg, uses a modified charge-pump circuit to deliver power from the low side to the high side.

\section{B. Operating Principles}

The operation of this new charge-pump circuit can be divided into the following three modes: charging, boost, and pumping modes. Each mode will be described individually assuming that the upper phase-leg switch is on. Under these conditions, the potential of the output node is nearly $V_{\mathrm{dc}}$. For convenience, the 


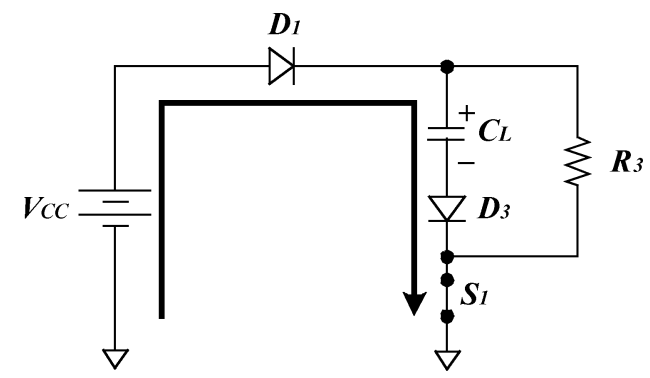

Fig. 2. Charging mode ( $S_{1}$ is on and $S_{2}$ is off).

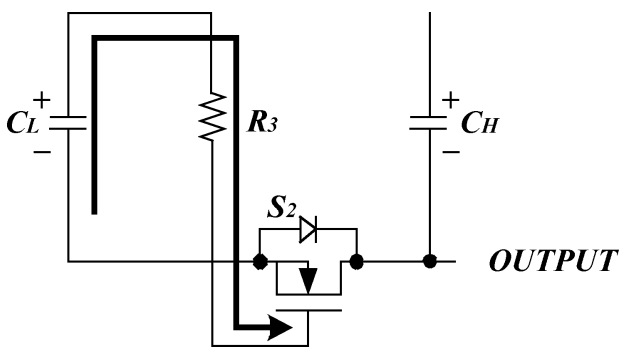

Fig. 3. Boost mode ( $S_{1}$ is off and $S_{2}$ is active).

negative rail is considered to be ground potential in the following description.

Charging Mode (Fig. 2): The boost capacitor $C_{L}$ in Fig. 1 is charged by the single low-side power supply $V_{\mathrm{CC}}$ through $D_{1}$ and $D_{3}$ when the switch $S_{1}$ turns on as shown in Fig. 2. $S_{2}$ and $D_{2}$ remain in their off-states since the gate-source voltage $V_{G S 2}$ is forced to $-V_{\mathrm{D} 3}$ during this charging mode. $D_{1}, D_{2}, S_{1}$ and $S_{2}$ must all be high-voltage devices that can sustain voltages up to the dc link voltage $V_{\mathrm{dc}}$. $D_{3}$ can be a low-voltage diode because its maximum reverse voltage is limited by the voltage on $C_{L}$ which is lower than $V_{\mathrm{CC}}$. A Schottky diode is preferred for $D_{3}$ in order to minimize its voltage drop and the resulting conduction losses.

Boost Mode (Fig. 3): When $S_{1}$ turns off, the voltage $V_{L}$ on the boost capacitor $C_{L}$ starts to charge the gate capacitance of $S_{2}, C_{\mathrm{GATE}}$, through $R_{3}$. Assuming that $C_{L}$ is much bigger than $C_{\mathrm{GATE}}$, the decrease in the $C_{L}$ voltage $V_{L}$ is negligible. The value of $R_{3}$ determines the turn-on time of $S_{2}$, but does not affect the magnitude of the final $S_{2}$ gate voltage. Once $C_{\mathrm{GATE}}$ is charged, there is no additional current flow or loss in $R_{3}$.

The voltage at the negative (lower) terminal of $C_{L}$ rises from ground level to $V_{\mathrm{dc}}$ (the output node voltage) as switch $S_{1}$ turns off and the drain-source voltage of $S_{2}$ decreases as shown in Fig. 3.

Pumping Mode (Fig. 4): After $S_{2}$ fully turns on, the charge in the boost capacitor $C_{L}$ is transferred to the high-side capacitor $C_{H}$ that serves as the local supply for the high-side switch gate. This pumping mode ends when $S_{1}$ is turned on again by the external control. Continuous switching of $S_{1}$ insures that gate drive charge is available at all times to the high-side switch without any interference with the desired phase-leg switching sequence.

\section{Paper Organization}

Models for each of the three modes are analyzed in Section II using linearized diode and metal oxide semiconductor field ef-

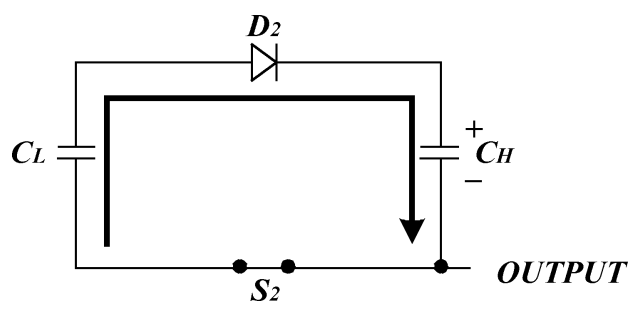

Fig. 4. Pumping mode ( $S_{1}$ is off and $S_{2}$ is on).
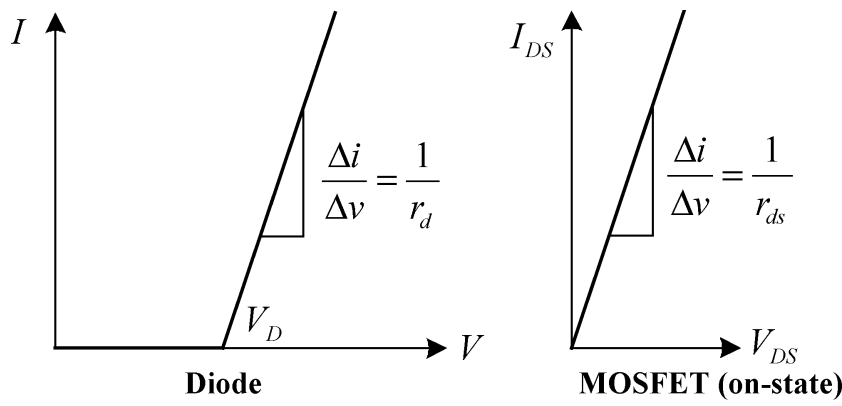

Fig. 5. Linearized diode and MOSFET models.

fect transistor (MOSFET) models that include the effects of load current. For high voltage applications, the voltage drops caused by the internal resistances of the diodes and MOSFETs become progressively more significant as their current levels increase. Steady-state operation is also analyzed, illuminating the effects of important parameters including frequency, duty cycle, delay times, and switching times. Following this analysis, simulation and experimental results are presented in Section III to verify the model and to demonstrate the circuit's operating characteristics.

\section{Charge Pump Circuit ANalysis}

Fig. 5 shows the voltage-current characteristics of a common piecewise-linear model for diodes and on-state MOSFETs that will be used in this simplified analysis. The V-I characteristics of the diode can be defined by two parameters - the voltage drop $V_{D}$ and the on-state resistance $r_{d}$.

As note above, a Schottky diode is a good choice for $D_{3}$ in order to minimize its forward voltage drop. The Schottky diode forward voltage is typically $0.1 \sim 0.2 \mathrm{~V}$, while the comparable voltage for a standard silicon junction diode is $0.7 \mathrm{~V}$.

The MOSFET will be modeled as a simple resistor $r_{d s}$ after it turns on as shown in Fig. 5. The reverse leakage current of the MOSFET and diode are ignored in this analysis. IGBT's could be used instead of MOSFETs for a high-voltage application $(>600 \mathrm{~V})$, but the forward voltage drop of an IGBT is typically higher than that of a MOSFET for low current conditions.

The voltage waveforms across each low-side and high-side capacitor during the three operating modes are shown in Fig. 6 for initial start-up operation. The fixed-frequency input control signal $V_{\mathrm{PULSE}}$ and the switch $S_{1}$ drain-source voltage $V_{\mathrm{DS} 1}$ are also shown to help explain the circuit's operation.

The equations for the boost capacitor $C_{L}$ voltage $V_{L}(t)$ and the high-side capacitor $C_{H}$ voltage $V_{H}(t)$ will be analyzed for each of the three operating modes using equivalent circuits. The value of $V_{H}(t)$ under steady-state conditions will also be calcu- 


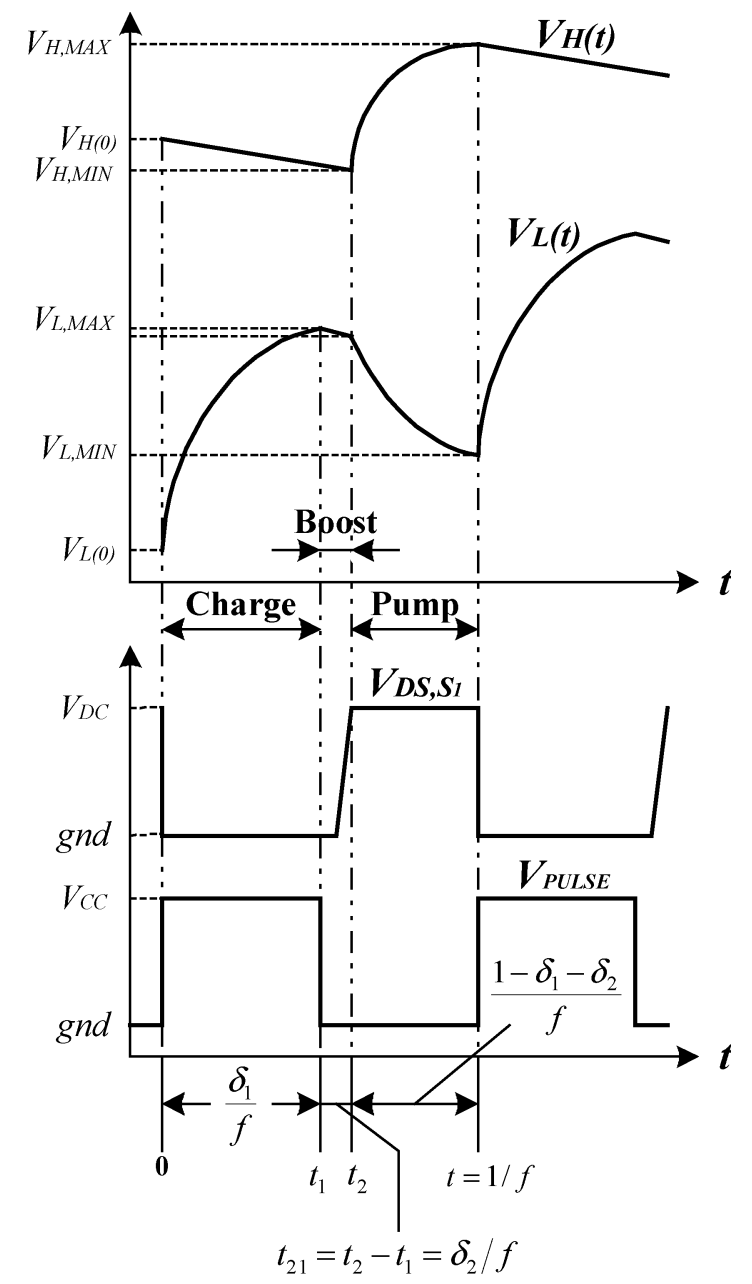

Fig. 6. Self-boost charge pump waveforms during initial start-up operation.

lated as a function of the boost time $t_{21}$ to illustrate the control principles for the new charge-pump circuit.

\section{A. Charging Mode Equivalent Circuit and Analysis}

During the charging mode, switch $S_{1}$ is on and switch $S_{2}$ is in its off state. The boost capacitor $C_{L}$ is charged through $D_{1}, D_{3}$ and $S_{1}$. The equivalent circuit for the charging mode is shown in Fig. 7. The maximum charging voltage of $C_{L}$ is $V_{\mathrm{L}, \mathrm{MAX}}=V_{\mathrm{CC}}-V_{\mathrm{D} 1}-V_{\mathrm{D} 3}$ and the equivalent resistor value is $R_{E Q}=r_{\mathrm{d} 1}+r_{\mathrm{d} 3}+r_{\mathrm{dS} 1}$. The boost capacitor voltage $V_{L}(t)$ during the charging mode can be calculated as

$$
v_{L}(t)=v_{L}(0)+\left[V_{\mathrm{L}, \mathrm{MAX}}-v_{L}(0)\right]\left(1-e^{\frac{-t}{R_{E Q} \cdot C_{L}}}\right) \text {. }
$$

It is worth noting that the same voltage $V_{\mathrm{L}, \mathrm{MAX}}$ (plus the $D_{3}$ diode drop) appears across resistor $R_{3}$ during the charging mode, resulting in power dissipation in $R_{3}$ during this interval.

\section{B. Boost Mode Equivalent Circuit and Analysis}

During the boost mode, $S_{1}$ turns off, $D_{3}$ transitions to its offstate, and the gate capacitance of $S_{2}$ is charged so $S_{2}$ begins to turn on. Fig. 8 shows the equivalent circuit during this operating mode. The time constant of the exponential voltage increase is

$$
\tau_{\text {Boost }}=R_{3} \frac{C_{L} C_{\mathrm{GATE}}}{C_{L}+C_{\mathrm{GATE}}}
$$

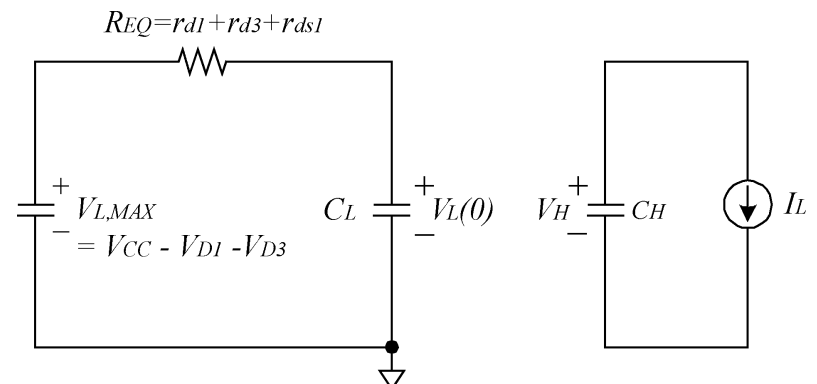

Fig. 7. Charging mode model.

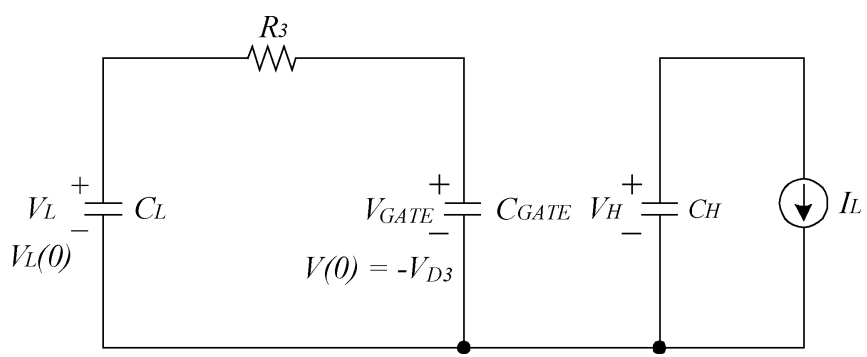

Fig. 8. Boost mode model.

where $C_{\mathrm{GATE}}$ is the total gate capacitance of $S_{2}$.

The voltage on both $C_{L}$ and $C_{\mathrm{GATE}}$ following completion of this gate-charging interval is

$$
V_{L}=\frac{C_{L} \cdot V_{L}(0)}{C_{L}+C_{\mathrm{GATE}}} .
$$

It is evident in (3) that if $C_{L}$ is much bigger than $C_{\mathrm{GATE}}$, the voltage decrease of $C_{L}$ compared to its initial value $V_{L}(0)$ is negligible, and the value of $R_{3}$ does not affect the final voltage value.

The load on the gate drive circuit is modeled by a constant load current $I_{L}$ that flows at all times. The floating high-side capacitor $C_{H}$ is discharged by this load current $I_{L}$ during both the charging and boost modes. Thus, the equation for the high-side capacitor voltage $V_{H}$ during the boost mode can be expressed simply as

$$
V_{H}(t)=V_{H}(0)-\frac{I_{L}}{C_{H}} \cdot t .
$$

\section{Pumping Mode Equivalent Circuit and Analysis}

The pumping mode begins when $S_{2}$ fully turns on while $S_{1}$ remains off. The equivalent circuit for this pumping mode is shown in Fig. 9, where the equivalent resistance $R_{\mathrm{EQ} 2}$ consists of the series combination of the equivalent resistances of diode $D_{2}\left(r_{\mathrm{d} 2}\right)$ and switch $S_{2}\left(r_{d s 2}\right)$. The high-side capacitor voltage $V_{H}$ can be derived as

$$
\begin{aligned}
& V_{H}(t)=V_{H}(0) e^{\frac{-t}{\tau}}+\frac{C_{L} V_{L}^{\prime}(0)+C_{H} V_{H}(0)}{C_{L}+C_{H}}\left(1-e^{\frac{-t}{\tau}}\right) \\
& -\frac{R_{\mathrm{EQ} 2} C_{L}^{2} I_{L}}{\left(C_{L}+C_{H}\right)^{2}}\left(1-e^{\frac{-t}{\tau}}\right)-\frac{I_{L}}{C_{L}+C_{H}} t \\
& \text { where } \quad \tau=R_{\mathrm{EQ} 2} \frac{C_{L} C_{H}}{C_{L}+C_{H}} \\
& R_{\mathrm{EQ} 2}=r_{\mathrm{d} 2}+r_{d s 2} \\
& V_{L}^{\prime}(0)=V_{L}(0)-V_{\mathrm{D} 2} \text {. }
\end{aligned}
$$




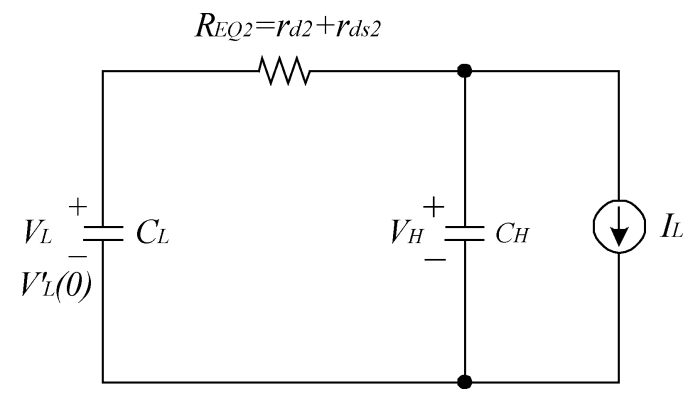

Fig. 9. Pumping mode model.

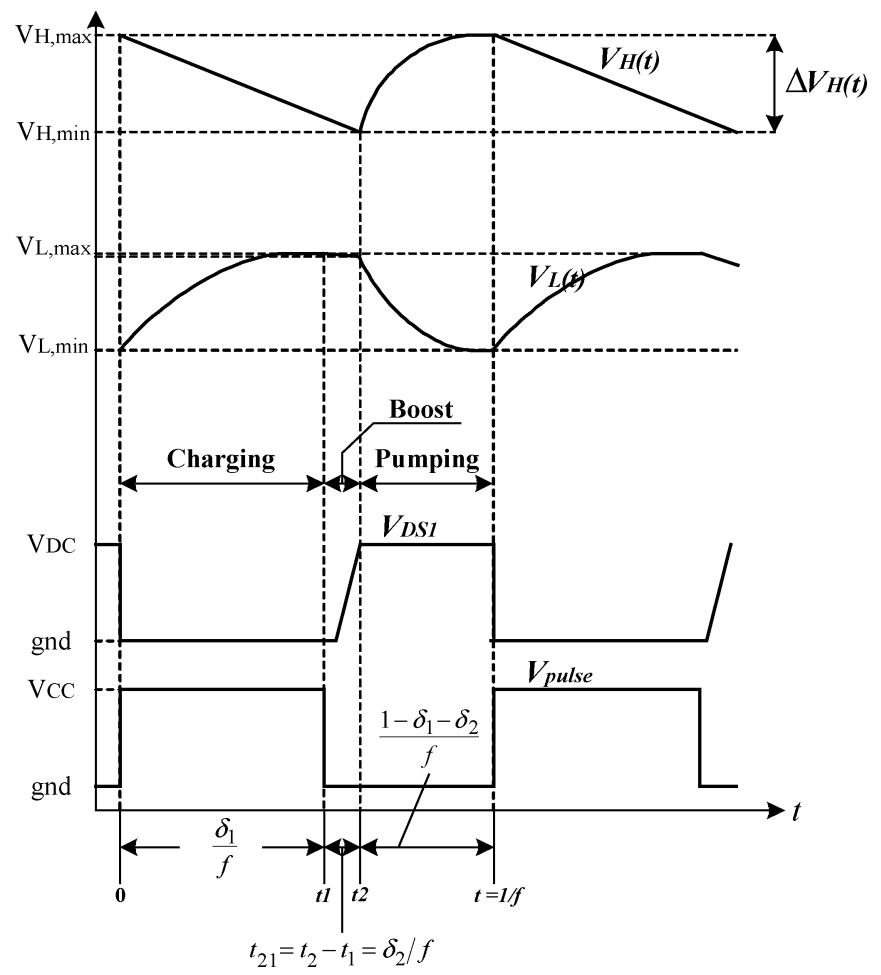

Fig. 10. Self-boost charge pump waveforms during steady-state operation.

The second term in (5) represents the stored capacitor charge in $C_{L}$ that is transferred from the low-side supply to the highside gate drive. The third and fourth terms represent the voltage drops due to the equivalent resistor and the gate drive load current, respectively.

\section{D. $V_{H}$ Under Steady-State Conditions}

Under steady-state conditions, the maximum and minimum voltage levels of $V_{H}$ and $V_{L}$ remain constant as shown in Fig. 10. Applying the steady-state constraints, the minimum value of the high-side capacitor voltage during steady-state conditions $V_{H, M I N}$ can be expressed as

$$
\begin{aligned}
& V_{H, M I N}=V_{\mathrm{H}, \mathrm{MAX}}-\Delta V_{H} \\
& \text { where } \Delta V_{H}=\frac{I_{L}}{C_{H}} \frac{\delta_{1}+\delta_{2}}{f}
\end{aligned}
$$

and the duty cycle values during the charging and boost intervals are $\delta_{1}$ and $\delta_{2}$, respectively (see Fig. 10).

In (9), the second term represents the ripple voltage amplitude $\Delta V_{H}$. In order to reduce the ripple voltage amplitude, either the value of the high-side capacitance $C_{H}$ must be increased or a higher frequency $f$ is required. However, the switching losses in switches $S_{1}$ and $S_{2}$ make it undesirable to raise the frequency too high. Reducing the duty cycle interval $\delta_{2}$ makes it possible to transfer more charge from the low to high sides, and this helps to reduce the ripple voltage amplitude. However, it is important to recognize that one of the most direct ways to reduce $\delta_{2}$ is to lower the value of resistor $R_{3}$ which causes higher power dissipation in $R_{3}$ during the charging mode. As a result, selection of the $R_{3}$ value represents of the significant tradeoffs that must be considered during the circuit design process.

$V_{\mathrm{H}, \mathrm{MAX}}$ can be derived starting from (5), defining the duration of the pumping interval as $t_{\mathrm{PUMP}}=\left(1-\delta_{1}-\delta_{2}\right) / f$ (see Fig. 10). If $t_{\mathrm{PUMP}}>2 \cdot \tau$, the value of the exponential function in this equation is less than $0.14\left(=e^{-2}\right)$. Assuming that this condition is met so that the terms proportional to this exponential can be ignored, (5) can be evaluated at the end of the pumping interval to establish an approximate expression for $V_{\mathrm{H}, \mathrm{MAX}}$

$$
\begin{aligned}
V_{\mathrm{H}, \mathrm{MAX}}= & V_{H}\left(t_{\mathrm{PUMP}}\right) \\
= & \frac{C_{L} V_{L}^{\prime}(0)+C_{H} V_{H}(0)}{C_{L}+C_{H}}-\frac{R_{\mathrm{EQ} 2} C_{L}^{2} I_{L}}{\left(C_{L}+C_{H}\right)^{2}} \\
& -\frac{I_{L}}{C_{L}+C_{H}} t_{\mathrm{PUMP}} .
\end{aligned}
$$

The final expression of $V_{\mathrm{H}, \mathrm{MAX}}$ can be derived using the following two initial conditions at the beginning of the pumping stage using the previously-defined variable $V_{\mathrm{L}, \mathrm{MAX}}$ and (9) and (10)

$$
\begin{aligned}
V_{L}^{\prime}(0) & =V_{\mathrm{L}, \mathrm{MAX}}-V_{\mathrm{D} 2} \\
& =V_{\mathrm{CC}}-V_{\mathrm{D} 1}-V_{\mathrm{D} 2}-V_{\mathrm{D} 3} \\
V_{H}(0) & =V_{H, M I N} \\
& =V_{\mathrm{H}, \mathrm{MAX}}-\frac{I_{L}}{C_{H}} \cdot \frac{\delta 1+\delta 2}{f} .
\end{aligned}
$$

Substituting these two expressions into (11) and solving for $V_{\mathrm{H}, \mathrm{MAX}}$ leads to

$V_{\mathrm{H}, \mathrm{MAX}}=V_{\mathrm{CC}}-V_{\mathrm{D} 1}-V_{\mathrm{D} 2}-V_{\mathrm{D} 3}-\frac{R_{\mathrm{EQ} 2} I_{L} C_{L}}{C_{L}+C_{H}}-\frac{I_{L}}{C_{L} f}$.

Equation (14) is valid only if the durations of the charging and pumping modes are sufficiently long compared to the $R C$ time constants during each of these intervals. If not, the maximum value of $V_{H}$ decreases and the exact value of $V_{\mathrm{H}, \mathrm{MAX}}$ should be calculated using (5). If possible, the time constants in (1) and (6) should be set to values that are small compared to the charging and pumping time intervals for a given frequency condition. Otherwise, the maximum output voltage decreases due to insufficient charging and pumping times. Although this suggests reduced values for $C_{L}$ and $C_{H}$, higher capacitance values are required to minimize the voltage ripple [see (10)] and to increase the maximum value of the output voltage $V_{\mathrm{H}, \mathrm{MAX}}$ in (14). This identifies another significant tradeoff that must be addressed during the circuit design process.

\section{E. Calculation of Boost Time $t_{21}$}

As the operating frequency $f$ increases, the effect of the boost time $t_{21}$ (see Fig. 6) on the output voltage is more significant be- 


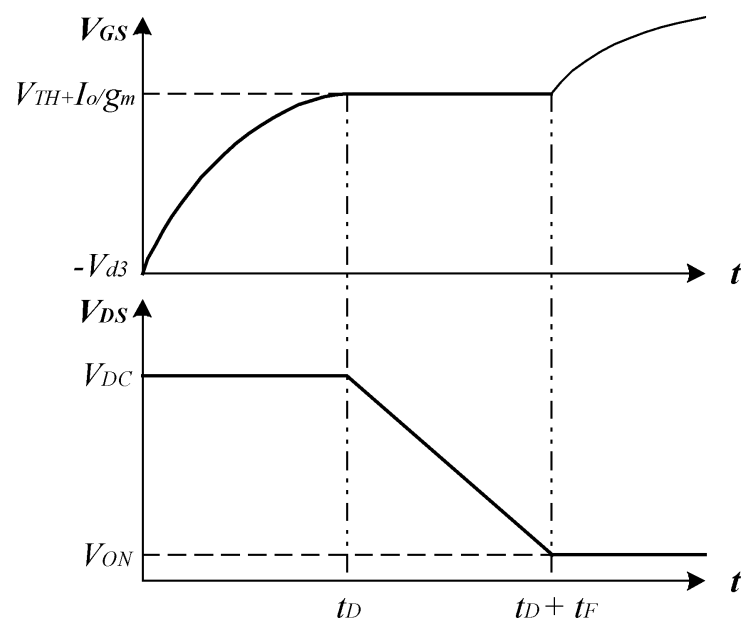

Fig. 11. $V_{\mathrm{GS}}$ and $V_{D S}$ waveforms for $S_{2}$ during $t_{21}$ boost interval.

cause the ratio of charging (pumping) to discharging time values for $C_{H}$ decreases if $t_{21}$ and duty cycle $\delta_{1}$ are fixed in value. It should be noted that $t_{21}$ is determined by the boost-mode time constant $\tau_{\text {Boost }}$ defined in (2). The duration of the boost mode does not change as the frequency $f$ is varied for a given switch $S_{2}$ and a given value of $R_{3}$. This makes $t_{21}$ a key parameter under higher frequency conditions since duty cycle $\delta_{2}$ is directly proportional to the frequency (i.e., $\delta_{2}=t_{21} * f$ ).

Closer examination reveals that the time interval $t_{21}$ can be separated into two subintervals consisting of a delay time $t_{D}$ and fall time $t_{F}$, representing the typical turn-on switching characteristics of $S_{2}$ as shown in Fig. 11. During $t_{D}$, the gate node is charged up to the threshold voltage $V_{\mathrm{TH}}$. At that point, the drain current starts to flow in $S_{2}$. $V_{D S}$ falls during $t_{F}$ while the gate-source voltage $V_{\mathrm{GS}}$ remains nearly constant at $V_{\mathrm{TH}}$ due to the Miller effect.

The intervals $t_{D}$ and $t_{F}$ can be calculated using the circuit and switch model parameters as follows [7]:

$$
\begin{aligned}
t_{D}= & R_{3} \cdot C_{\mathrm{EQ} 2} \cdot \ln \frac{V_{\mathrm{CC}}-V_{\mathrm{D} 1}-V_{\mathrm{D} 2}}{V_{\mathrm{CC}}-V_{\mathrm{D} 1}-2 \cdot V_{\mathrm{D} 2}-V_{\mathrm{TH}}-\frac{g_{m}}{I_{O}}} \\
& +R_{3} \cdot C_{\mathrm{EQ} 2} \cdot \ln \frac{V_{\mathrm{CC}}-V_{\mathrm{D} 1}-V_{\mathrm{D} 2}}{V_{\mathrm{CC}}-V_{\mathrm{D} 1}-V_{\mathrm{D} 2}-V_{\mathrm{TH}}} \\
t_{F}= & \frac{V_{\mathrm{dc}} \cdot R_{3} \cdot C_{\mathrm{GD}}}{V_{L(0)}-V_{\mathrm{TH}}-\frac{g_{m}}{I_{O}}}
\end{aligned}
$$

where

$V_{\text {TH }}$ gate threshold voltage $[V]$;

$V_{\text {dc }} \quad$ dc link voltage $[V]$;

$g_{m} \quad$ device transconductance $[A / V]$;

$I_{O} \quad$ load inductor current $[A]$

$$
C_{\mathrm{EQ} 2}=\frac{C_{L} \cdot\left(C_{\mathrm{GD}}+C_{\mathrm{GS}}\right)}{C_{L}+C_{\mathrm{GD}}+C_{\mathrm{GS}}}[F]
$$

$C_{\mathrm{GD}}$ device gate-drain capacitance $[F]$;

$C_{\mathrm{GS}}$ device gate-source capacitance $[F]$.

It should be no surprise that choosing a MOSFET for $S_{2}$ that has fast switching characteristics, including low values of $C_{\mathrm{GD}}$ and $C_{\mathrm{GS}}$, helps to reduce the duration of the boost interval

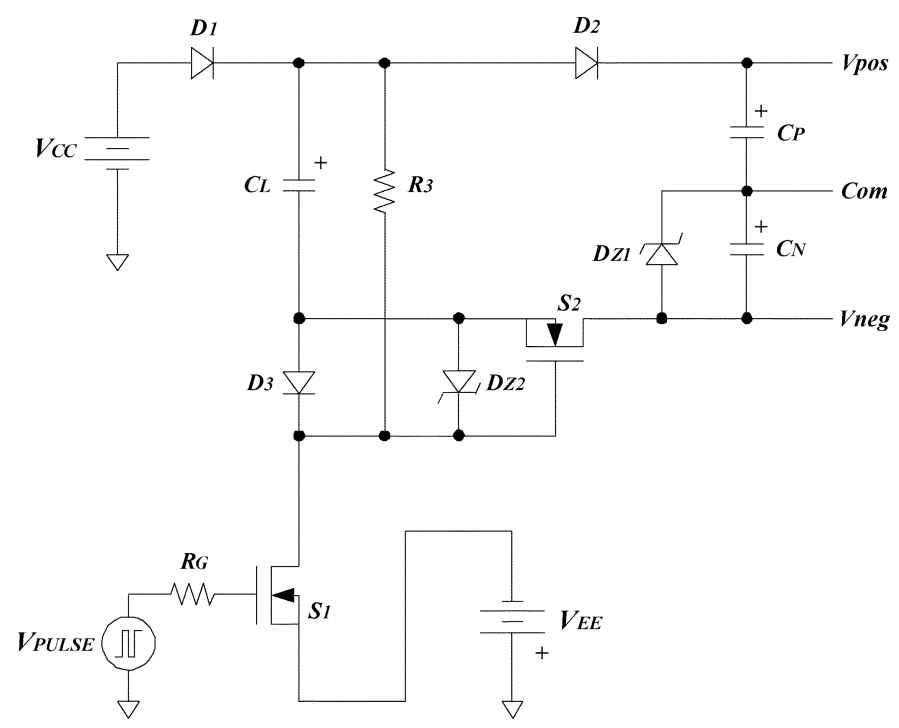

Fig. 12. Modified self-boost charge pump with positive- and negative-bias voltage supply capability.

$t_{21}$ by reducing the delay and fall times $t_{D}$ and $t_{F}$. In addition, (15)-(17) indicate that reducing the values of $R_{3}$ and $C_{L}$ also help to reduce $t_{21}$. However, the performance tradeoffs involved in lowering the values of these two circuit component values have already been noted and will be discussed further in Section II-G.

\section{F. Circuit Modifications for Bipolar Gate Voltages}

Negative gate bias is widely used in high-power phase-leg applications in order to prevent dangerous retriggering problems. Fig. 12 shows a modified version of the self-boost charge pump scheme with positive and negative voltage supply capability. Zener diode $D_{\mathrm{Z} 1}$, positive voltage capacitor $C_{P}$, and negative voltage capacitor $C_{N}$ are used to split the boost capacitor voltage $V_{L}$ into two parts for the high-side gate drive. The second supply voltage $V_{\mathrm{EE}}$ is added to provide negative gate voltage for the low-side switch in the phase leg. The ground terminal in this figure corresponds to the low-side dc bus voltage, consistent with Fig. 1.

During the charging mode, boost capacitor $C_{L}$ is charged by $V_{\mathrm{CC}}+V_{\mathrm{EE}}$ in order to develop nearly the same positive and negative voltages ( $V_{\mathrm{CC}}$ and $V_{\mathrm{EE}}$, respectively) in the high-side gate drive. The maximum charging voltage of $C_{L}$ is $V_{\mathrm{L}, \mathrm{MAX}, \mathrm{PN}}=$ $V_{\mathrm{CC}}+V_{\mathrm{EE}}-V_{\mathrm{D} 1}-V_{\mathrm{D} 3}$. Zener diode $D_{\mathrm{Z} 2}$ is required to insure that the maximum safe gate-source voltage of $S_{2}$ is not exceeded during the boost or pumping modes. During the pumping mode, stored charge in $C_{L}$ is distributed to both $C_{P}$ and $C_{N}$, with the negative-bias voltage developed across $C_{N}$ limited by $D_{\mathrm{Z} 1}$.

The zener diode $D_{\mathrm{Z} 1}$ should be selected with a clamping voltage $V_{\mathrm{DZ} 1}$ that is less than $V_{\mathrm{EE}}$ in order to maintain a stable negative bias voltage source for the high-side switch under all operating conditions. If the clamping voltage $V_{\mathrm{DZ} 1}$ is higher than $V_{\mathrm{EE}}$, the negative-bias gate voltage for the high-side switch will drop to the lower voltage $V_{\mathrm{EE}}$ when the low-side switch is on. This occurs because the Com terminal in Fig. 12, corresponding to the low-side switch collector voltage (see Fig. 1), 
falls to nearly ground (i.e., low-side dc bus) potential when the low-side switch is on.

Using (14), approximate expressions for the negative-bias voltage $V_{N}$ and the maximum positive-bias voltage $V_{\mathrm{P}, \mathrm{MAX}}$ under steady-state conditions can be derived as

$$
\begin{aligned}
V_{N}= & V_{\mathrm{DZ} 1} \\
V_{\mathrm{P}, \mathrm{MAX}}= & V_{\mathrm{CC}}+V_{\mathrm{EE}}-V_{\mathrm{DZ} 1}-V_{\mathrm{D} 1}-V_{\mathrm{D} 2}-V_{\mathrm{D} 3} \\
& -\frac{R_{\mathrm{EQ} 2} I_{L} C_{L}}{C_{L}+C_{H}}-\frac{I_{L}}{C_{L} f} .
\end{aligned}
$$

\section{G. Circuit Design Guidelines}

In order to minimize cost and complexity, the high-side power supply uses a simple open-loop control to set the output voltage with a fixed-frequency, fixed duty-cycle switching sequence. The desired output voltage characteristics can be specified in terms of the maximum output voltage and ripple voltage amplitude for a specified load current. These target values for $V_{\mathrm{H}, \mathrm{MAX}}, \Delta V_{H}$, and $I_{L}$ provide the necessary starting points for determining values for the circuit components.

Once these three performance parameters are set, tradeoffs between the sizes of the circuit components (influencing cost) and the circuit losses come to the forefront in determining the circuit component values. This tradeoff is particularly apparent when considering the voltage ripple requirement. Equation (10) indicates that product of $C_{H}$ and frequency $f$ must be approximately constant in order to achieve a particular value of ripple voltage $\Delta V_{H}$ for the specified load current $I_{L}$. (It is assumed that the sum of the duty cycles $\left(\delta_{1}+\delta_{2}\right)$ can vary over only a rather narrow range in the vicinity of 0.5 .) Increasing capacitance $C_{H}$ increases the capacitor's size and cost, while raising the frequency $f$ increases the circuit losses because of higher total switching losses in $S_{1}$ and $S_{2}$.

In addition, increasing frequency $f$ will likely make it necessary to reduce the value of resistor $R_{3}$, further increasing circuit losses, as described previously. As a general guideline, the value of $R_{3}$ should be chosen so that the boost interval duty cycle $\delta_{2}$ does not exceed 0.1 at the desired operating frequency. Higher values of $\delta_{2}$ are likely to unacceptably degrade the charging times for capacitors $C_{L}$ and $C_{H}$ during the charging and pumping modes, respectively. The average power loss in $R_{3}$ can be calculated approximately by assuming a $50 \%$ duty cycle (i.e., $\delta_{1}=0.5$ ), leading to the following expression:

$$
P_{R 3}=\frac{\left(V_{\mathrm{CC}}-V_{\mathrm{D} 1}\right)^{2}}{2 \cdot R_{3}} .
$$

Another factor that must be considered in the process of selecting the operating frequency is the quality of the MOSFET switches $S_{1}$ and $S_{2}$. Better MOSFETs with lower values of on-state resistance $r_{d s}$, lower gate capacitance [ $C_{\mathrm{GATE}}$ in (2)], and faster switching speeds will reduce circuit losses and make it considerably easier to raise the frequency $f$. However, adoption of such improved devices is likely to increase the MOSFET size and cost.

Ultimately, selection of the frequency depends on the application. For example, if the target application calls for integrating the high-side power supply inside a power module, then minimizing the volume of the capacitor components is likely to be a high priority. In this case, the design approach would likely call for raising the operating frequency as high as possible, limited by the maximum losses that can be safely dissipated inside the module.

Up to this point, it has been assumed that the value of the charging mode duty cycle $\delta_{1}$ is 0.5 . This is a reasonable choice because of the need to charge and discharge low-side capacitor $C_{L}$ each cycle. In fact, a simple timer circuit may force $\delta_{1}$ to be fixed at 0.5. However, further tuning the value of $\delta_{1}$ (when practical) may yield some improvement in circuit performance by providing a means to compensate for the impact of the boost duty cycle $\delta_{2}$ and differences between the time constants for the charging and pumping intervals [see (1) and (5)].

The selection of capacitance of $C_{L}$ also involves tradeoffs between competing objectives of performance and size/cost. In particular, increasing capacitance $C_{L}$ tends to increase the peak value of the output voltage $V_{\mathrm{H}, \mathrm{MAX}}$ for a given value of supply voltage $V_{\mathrm{CC}}$ because of the importance of the last term in (14). The penalty is a larger capacitor. Considering these tradeoffs, a good starting point is to choose $C_{L}$ and $C_{H}$ to be equal in value, resulting in time constants for the $C_{L}$ charging and discharging (i.e., pumping) intervals that have similar values.

Assuming that the conditions are met for (14) to give a good approximation of $V_{\mathrm{H}, \mathrm{MAX}}$, (14) can be used to eliminate load current $I_{L}$ and frequency $f$ from the expression. If one accepts the observation that the next-to-last term in (14) can typically be ignored because it is small compared to all of the others, the equation can be rearranged to yield an approximate expression for the supply voltage $V_{\mathrm{CC}}$ needed to achieve the specified $V_{\mathrm{H}, \mathrm{MAX}}$ value

$$
V_{\mathrm{CC}} \cong V_{\mathrm{H}, \mathrm{MAX}}+V_{\mathrm{D} 1}+V_{\mathrm{D} 2}+V_{\mathrm{D} 3}+\frac{C_{H}}{C_{L}} \frac{\Delta V_{H}}{\left(\delta_{1}+\delta_{2}\right)} .
$$

\section{Simulation AND EXPERIMENTAL RESUlts}

Simulations and experimental tests were carried out to verify the operation of the self-boost charge pump circuit. Fig. 13 shows the test circuit that was used for the laboratory tests. The simulations have been carried out using PSPICE with the same test circuit. The devices used for the switches $S_{1}$ and $S_{2}$ are both the same MOSFET type BUZ50B manufactured by Infineon and rated at $1000 \mathrm{~V}$ and $3 \mathrm{~A}$. Diode types $1 \mathrm{~N} 4007$ and 1 N5818 were selected for the high-voltage diode $D_{2}$ and the low-voltage Schottky diode $D_{3}$, respectively. The reverse blocking voltage of the $1 \mathrm{~N} 4007$ diode is $1000 \mathrm{~V}$, the same as the MOSFET voltage rating.

The test conditions used for both the experimental tests and the simulations are as

$$
\begin{array}{llll}
V_{\mathrm{dc}}=600 \mathrm{~V} & V_{\mathrm{CC}}=20 \mathrm{~V} & f=5 \mathrm{kHz} & \delta_{1}=0.5 \\
C_{L}=10 \mu \mathrm{F} & C_{H}=10 \mu \mathrm{F} & R_{g}=2 \mathrm{k} \Omega & R_{o}=600 \Omega . \\
R_{3}=2 \mathrm{k} \Omega & & &
\end{array}
$$

The value of $R_{o}$ was set to $600 \Omega$ to adjust the load current to approximately $28 \mathrm{~mA}$ for a $V_{H}$ value of $17 \mathrm{~V}$. This resistor represents an average load of $476 \mathrm{~mW}$ imposed by the high-side switch gating. For reference, this power level would be sufficient for use with commercially-available gate drive ICs to drive a 


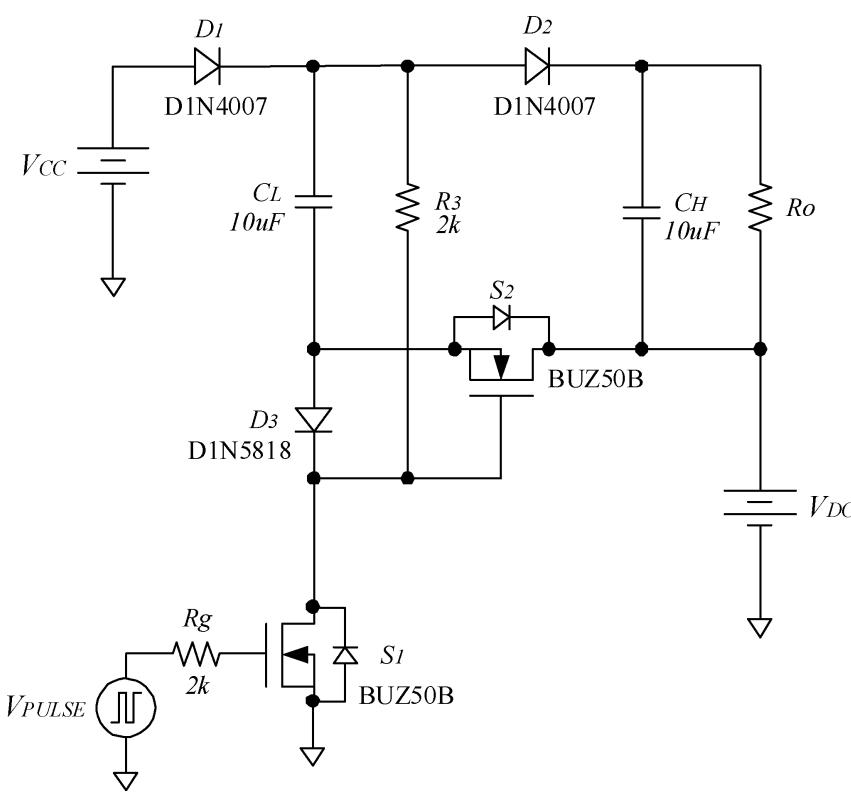

Fig. 13. Self-boost charge pump test circuit.

$1200-V$ IGBT with a $100-A$ current rating at frequencies in the 10-20 $\mathrm{kHz}$ range [8].

The ripple voltage can be calculated from (10)

$$
\Delta V_{H}=\frac{I_{L}}{C_{H}} \frac{\delta 1+\delta 2}{f}=\frac{0.028 *(0.6)}{(10 e-6 * 5000)}=0.34 \mathrm{~V} \text {. }
$$

The sum of the charging- and boost-mode duty cycle values $\left(\delta_{1}+\delta_{2}\right)$ is assumed to be 0.6 (i.e., $\left.\delta_{2}=0.1\right)$ based on the selected circuit design values.

Rearranging (21), the maximum high-side output voltage $V_{\text {H,MAX }}$ can be approximately calculated to be

$$
\begin{aligned}
V_{\mathrm{H}, \mathrm{MAX}} & \cong V_{\mathrm{CC}}-V_{\mathrm{D} 1}-V_{\mathrm{D} 2}-V_{\mathrm{D} 3}-\frac{C_{H}}{C_{L}} \frac{\Delta V_{H}}{\left(\delta_{1}+\delta_{2}\right)} \\
& =20-0.8-0.8-0.2-(1.67 * 0.34) \\
& =17.6 \mathrm{~V}
\end{aligned}
$$

where it is assumed that the $V_{\mathrm{D} 1}=V_{\mathrm{D} 2}=0.8 \mathrm{~V}$ and $V_{\mathrm{D} 3}=$ $0.2 \mathrm{~V}$ because $D_{1}$ and $D_{2}$ are high-voltage diodes and $D_{3}$ is a Schottky diode.

Fig. 14 shows experimental waveforms and overlaid simulation results for the input control voltage $V_{\text {PULSE }}$, the high-side capacitor voltage $V_{H}$, and the switch $S_{1}$ drain-source voltage $V_{\mathrm{DS} 1}$ under steady-state conditions. The measured value of the ripple voltage $\Delta V_{H}$ is approximately $0.4 \mathrm{~V}$ which is small enough to insure robust performance of the high-side gate drive.

The calculated maximum capacitor voltage $V_{\mathrm{H}, \mathrm{MAX}}$ and ripple voltage $\Delta V_{H}$ values demonstrate satisfactory agreement with the measured values $\left(V_{\mathrm{H}, \mathrm{MAX}-\text { Meas }}=17.6 \mathrm{~V}\right.$ and $\Delta V_{H-\text { Meas }}=0.45 \mathrm{~V}$ ). The simulated and experimental waveshapes also exhibit good agreement, although the measured $V_{H}$ waveform in Fig. 14 exhibits a small sudden downward step at the beginning of the charging mode that is not predicted by the simple analysis presented above. The amplitude of this step is approximately $0.1 \mathrm{~V}$, nearly equal to the difference between the calculated and measured values of $\Delta V_{H}$. Investigation has revealed that this voltage step is caused by the reverse recovery
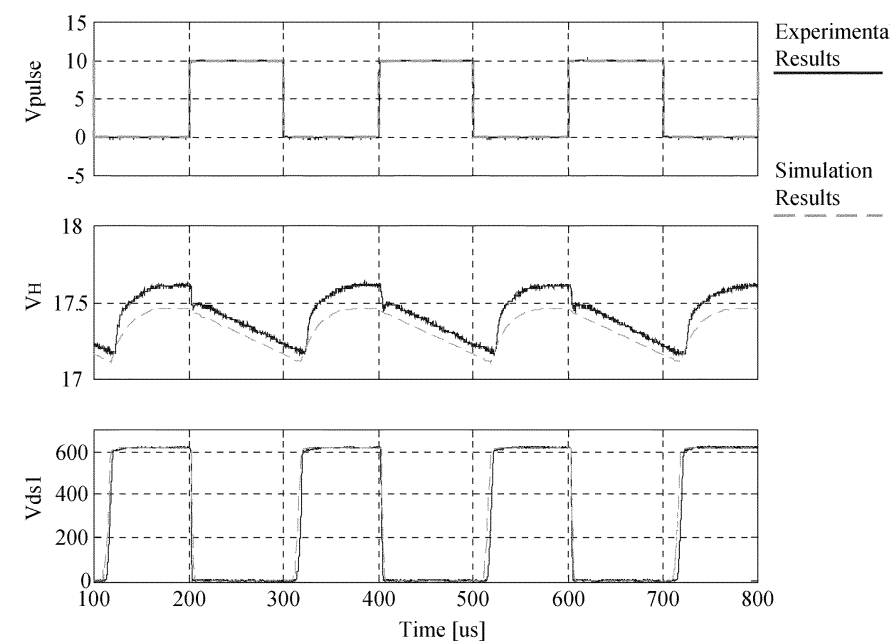

Fig. 14. Simulation and experimental results for $V_{\mathrm{PULSE}}, V_{H}$, and $V_{\mathrm{DS} 1}$.

current of diode $D_{2}$ when $S_{1}$ turns on. Stored charge in $C_{H}$ is transferred to $C_{L}$ during the $D_{2}$ reverse recovery interval, resulting in the observed voltage step. This diode reverse recovery characteristic is not been included in the simulation model, so the voltage step does not appear in the simulated $V_{H}$ waveform in Fig. 14.

The value of the $S_{1}$ turn-on interval $t_{21}$ can be measured directly from the experimental waveforms in Fig. 14 to be approximately $20 \mu$ s. Even though the duty cycle of $V_{\text {PULSE }}$ (i.e., $\delta_{1}$ ) is set at 0.5 , the actual duty cycle of the $C_{H}$ charging interval $\left(=1-\delta_{1}-\delta_{2}\right)$ is approximately 0.4 , meaning that the $t_{21}$ boost-mode interval reduces the charging duty cycle by approximately $20 \%$ (from 0.5 to 0.4 ). Reducing the value of $\delta_{2}$ would cause the ripple voltage amplitude $\Delta V_{H}$ to decrease, but the penalty of higher losses in resistor $R_{3}$ must be considered as discussed previously in Section II. The average power dissipation in resistor $R_{3}$ is calculated to be approximately $92 \mathrm{~mW}$ using (20) compared to the delivered high-side output power of $476 \mathrm{~mW}$.

\section{CONCLUSION}

This paper has described a new self-boost charge pump topology for a floating high-side gate drive power supply. This new power supply circuit provides the following attractive features compared to conventional techniques:

1) robust gate drive power availability for high-side switch that eliminates phase-leg refresh switching requirements even if the high-side switch is permanently on;

2) simple charge-pump configuration requiring no high-frequency magnetic components;

3) high steady-state current supply capability for high-performance gate drives;

4) configurable to provide bipolar gate source voltages for high-side switch;

5) compatible with integrated circuit implementation.

A model for each circuit mode has been derived using piecewise-linear device models. Taken together, these models provide useful predictions of both steady-state and transient oper- 
ating characteristics, as well as valuable insights regarding design considerations. Both simulation and experimental results have been presented to confirm the promising operating characteristics of the self-boost charge pump circuit. Guidelines have been presented to provide assistance with selection of circuit component values for applying this high-side power supply circuit to new applications.

\section{REFERENCES}

[1] HW floating MOS-gate drive ICs, in IR Application Note AN-978, International Rectifier Corp., El Segundo, CA.

[2] S. Storti, F. Consiglieri, and M. Paparo, "A 30 A 30 V DMOS motor controller and driver," IEEE J. Solid-State Circuits, vol. 23, no. 12, pp. 1394-1401, Dec. 1988.

[3] G. D. Cataldo and G. Palumbo, "Double and triple charge pump for power IC: dynamic models which take parasitic effects into account," IEEE Trans. Circuits Syst., vol. 40, no. 2, pp. 92-101, Feb. 1993.

[4] R. L. Lin and F. C. Lee, "Single-power-supply-based transformerless IGBT/MOSFET gate driver with 100\% high-side turn-on duty cycle operation performance using auxiliary bootstrapped charge pumper," in Proc. IEEE Power Electronics Specialties Conf. (PESC), Jun. 1997, pp. 1205-1209.

[5] G. F. W. Khoo, D. R. H. Carter, and R. A. McMahon, "Analysis of a charge pump power supply with a floating voltage reference," IEEE Trans. Circuits Syst., vol. 47, no. 10, pp. 1494-1501, Oct. 2000.

[6] - "Comparison of charge pump circuits for half-bridge inverters," Proc. Inst. Elect. Eng., vol. 147, pp. 356-362, Dec. 2000.

[7] B. J. Baliga, Power Semiconductor Devices. Boston, MA: PWS, 1995, pp. 387-397.

[8] Agilent Technologies, "2.0 amp gate drive optocoupler with integrated desaturation detection and fault status feedback," HCPL-316J Data Sheet, 1999.

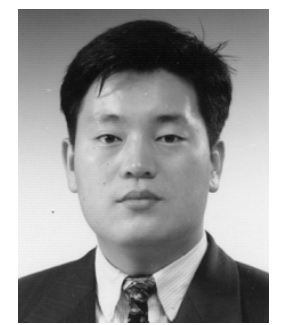

Shihong Park (S'01) received the B.S. degree in electrical engineering from Yonsei University, Seoul, Korea, in 1988, and the M.S. and Ph.D. degrees from the University of Wisconsin, Madison, in 2003 and 2004, respectively.

From 1988 to 1998, he was with Samsung Electronics, Buchen, Korea, as a Senior Power IC Design Engineer. In 2004, he was a Principal Research Engineer with Fairchild Semiconductor Korea, Buchen, Korea. In March 2005, he joined the School of Electrical, Electronics, and Computer Engineering, Dankook University, Seoul. His main research interests are integrated power electronics modules (IPEMs), power ICs and devices for automotive applications, active gate drive topology, and power converter circuit.

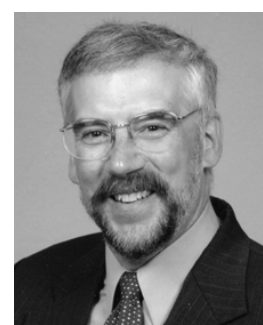

Thomas M. Jahns (F'93) received the S.B. and S.M., and Ph.D., degrees from the Massachusetts Institute of Technology (MIT), Cambridge, in 1974 and 1978, respectively, all in electrical engineering.

$\mathrm{He}$ joined the Faculty of the University of Wisconsin-Madison (UW-Madison) in 1998 as a Grainger Professor of Power Electronics and Electric Machines in the Department of Electrical and Computer Engineering. He is an Associate Director of the Wisconsin Electric Machines and Power Electronics Consortium (WEMPEC). Prior to coming to UW-Madison, he worked at GE Corporate Research and Development (now GE Global Research Center), Schenectady, NY, for 15 years, where he pursued new power electronics and motor drive technology in a variety of research and management positions. His research interests include permanent magnet synchronous machines for a variety of applications ranging from high-performance machine tools to low-cost appliance drives. From 1996 to 1998 , he conducted a research sabbatical at MIT, where he directed research activities in the area of advanced automotive electrical systems and accessories as co-director of an industry-sponsored automotive consortium.

Dr. Jahns received the William E. Newell Award from the IEEE Power Electronics Society (PELS) in 1999. He has been recognized as a Distinguished Lecturer by the IEEE Industry Applications Society (IAS) from 1994 to 1995 and by IEEE-PELS from 1998 to 1999 . He has served as President of PELS (1995-1996) and as a Member of the IAS Executive Board from 1992 to 2001. From 2002 to 2003, he was the Division II Director on the IEEE Board of Directors. 